\title{
BOOK REVIEW- ESTABLISHING THE EFFICACY OF TELEMEDICINE AS A CLINICAL TOOL FOR PHYSIOTHERAPISTS: FROM SYSTEMS DESIGN TO RANDOMISED CONTROLLED TRIAL
}

\author{
DANIEL G. RENDEIRO, PT, DSC, OCS, FAAOMPT \\ OCCUPATIONAL AND PHYSiCAL THERAPY SERVICE, WARrior Transition UNIT, Fort HOOD, TEXAS, USA
}

\section{ABSTRACT}

Trevor G. Russell's 2010 book, Establishing the Efficacy of Telemedicine as a Clinical Tool for Physiotherapists: From Systems Design to Randomised Controlled Trial, is based on his University of Queensland doctoral thesis that systematically demonstrates the feasibility of telemedicine for physical therapists. The book sets forth a highly effective answer to skeptics on this point, especially in the context of the traditionally "hands-on" subset of physical therapy (PT) represented by supervised postoperative physical rehabilitation. Russell has included much specific detail, such that clinicians, information managers, and logisticians planning the rollout of a telehealth system could streamline that process by referring to this important guide.

Key words: physical rehabilitation, physical therapy, physiotherapy, telehealth, telemedicine, telerehabilitation

Book reviewed: Russell, T. (2010). Establishing the efficacy of telemedicine as a clinical tool for physiotherapists: From systems design to randomised controlled trial. Saarbrücken, Germany: Lambert Academic Publishing.

Trevor G. Russell's 2010 book, Establishing the efficacy of telemedicine as a clinical tool for physiotherapists: From systems design to randomised controlled trial, is based on his University of Queensland doctoral thesis that systematically demonstrates the feasibility of telemedicine for physical therapists. It is a highly effective answer to skeptics on this point, especially in the context of the traditionally "hands-on" subset of physical therapy (PT) represented by supervised postoperative physical rehabilitation. The author describes in detail how a telemedicine system for PT can be imagined, validated, and launched, and he shows the reliability, validity, and clinical utility of each of its components.

The well-illustrated text includes tables and charts to guide the reader through in-home assessment, rehabilitation, and re-assessment of patients following knee replacement surgery-all via telehealth technology-to show how it can work. The author includes all major steps, from brainstorming, to pilot testing, to full implementation of the requisite system that includes the hardware, the software, and "user interface" between the therapist and patient. Cost and practicality are at the forefront of the discussion, inasmuch as one of the strategic goals of telehealth systems is to contain costs.

By focusing on a diagnosis that is readily recognizable as high-volume, Russell shows how a telemedicine approach has immense potential. Russell offers a convincing case that via use of this technology to leverage skilled expertise, healthcare systems can effectively serve populations that might be traditionally underserved, and deliver care more safely and economically to individuals who are increasingly likely to be aging and less mobile in the community.

This book mentions but does not explore in detail the use of telehealth systems for delivery of non-traditional PT care. There is substantial potential for using this modality for motivational interviews to initiate new behaviors; to check, encourage, and reinforce longterm compliance with specific rehabilitation protocols; and to prevent disability by promoting general physical exercise programs. The author's mechanistic approach omits examples of human interest in the context of how a specific person or people experienced benefits from a telemedicine approach-or what might be lost in the shift away from in-person care. Several target audiences would find this manuscript well worth reading. Chief among them are clinician-scientists. Russell's methodology qualifies as the highest level of evidence any single study can constitute--a randomized controlled trial. Policymakers and healthcare enterprise administrators 
with less scientific backgrounds, however, would also find this text to be useful in learning the true potential of what telemedicine systems can accomplish.

Finally, the document includes so much specific detail that clinicians, information managers, and logisticians planning the rollout of a telehealth system could streamline that process by referring to this important guide. Computer and video conferencing hardware have certainly changed to some degree in the years since the author conducted his work, but the principles of data capture and analysis, and patient assessment and education from a distance, remain the same.

\section{REFERENCES}

1. Russell, T. (2004). Establishing the efficacy of telemedicine as a clinical tool for physiotherapists: From systems design to randomised controlled trial (PhD thesis). University of Queensland, Brisbane, Australia.

2. Russell, T. (2010). Establishing the efficacy of telemedicine as a clinical tool for physiotherapists: From systems design to randomised controlled trial. Saarbrücken, Germany: Lambert Academic Publishing. 